\title{
Introducing a formal night-to-morning handover on the acute medical unit Princess Royal University Hospital (PRUH)
}

\author{
Authors: Amanda Mootoo ${ }^{A}$ and Harry Wright ${ }^{A}$
}

\section{Background}

Handover allows responsibility for immediate and continued care to be transferred between healthcare professionals. The National Confidential Enquiry into Patient Outcome and Death and the Royal College of Physicians identified handover as a high-risk step of the patient pathway. ${ }^{1}$ Thus, lack of handover such as that at Princess Royal University Hospital (PRUH) is a major preventable cause of patient harm and critical to patient safety.

\section{Aim}

The aim was to develop a formal, structured AMU night-tomorning handover, to enable safe recognition and handover of unstable and unwell patients, and to highlight priority tasks and specialty referrals.

\section{Method: Defining the problem}

A qualitative questionnaire was sent to all PRUH acute medical unit (AMU) junior doctors and consultants via online survey.

$100 \%$ of respondents defined current handover practice as unsafe and $100 \%$ identified need for morning handover. Handing over unwell patients in a timely and reliable manner to relevant team was raised as a primary issue. A 15 -minute (100\%) morning handover, led by registrars $(80 \%)$ involving night and day junior doctors (95\%) and AMU consultants (75\%) was felt best placed to address the issue.

\section{Cycle 1}

Interventions

Introduction of daily verbal and written handover using a formatted whiteboard, which identified all staff members, highlighted any unwell patients, priority tasks and potential staffing issues.

Use of a consultant cue-card to standardise the handover.

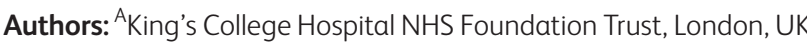

\section{Feedback}

Informal verbal feedback post cycle 1 - highlight overnight adverse incidents, hospital operational concerns and giving a learning point of the day to morning team.

Cycle 2

Interventions

Improved visual clarity of whiteboard and development of laminated handover cue-cards produced and printed to guide succinct handover delivery by night team.

\section{Remeasure}

Repeat online survey re-sent to all junior doctors and consultants on AMU 6 months post cycle 2 interventions. Overall positive response to introduction of AMU morning handover: $90 \%$ agreed new handover safely identifies unwell patients to day team; $50 \%$ improvement in handing over to the relevant team; $40 \%$ suggested an electronic handover would be useful.

\section{Cycle 3}

Development of an electronic handover request. This provides formal electronic log of handover, enables night team to document accurate details to aid morning handover.

\section{Sustainability}

We plan to repeat further cycles and re-assess interventions, to appoint a handover champion, to embed the handover within inductions, to collect feedback from consultants and to involve senior nursing staff and hospital managers.

\section{Strengths and limitations}

It enabled the introduction and implementation of a simple, cost-effective, formal AMU morning handover at PRUH that follows elements of the BM]'s 'good handover' practice. It has shown demonstrable improvement in addressing trainees' concerns. It follows the structure of a quality improvement project and involved three cycles with interventions reviewed 
after each cycle. Direct impact on patient safety was not measured as it did not use patient safety outcome measures.

\section{Conclusion}

Handover should be a priority given it is a high-risk step in the patient pathway. This project achieved the introduction and sustainment of a standardised night-to-morning AMU handover, which has undoubtedly had a positive role in improving patient safety by more efficient handover of unwell patients and has addressed concerns of the healthcare team.

\section{Conflicts of interest}

None declared.

\section{Reference}

1 Royal College of Physicians. Acute care toolkit 1: Handover. London: RCP, 2011. www.rcplondon.ac.uk/guidelines-policy/acute-caretoolkit-1-handover [Accessed 14 March 2019]. 